\title{
BMJ Open Prospective repeated assessment of self- reported sleep quality and sleep disruptive factors in the intensive care unit: acceptability of daily assessment of sleep quality
}

Ghaida Alsulami, ${ }^{1,2}$ Ann Marie Rice, ${ }^{3}$ Lisa Kidd ${ }^{4}$

To cite: Alsulami G, Rice AM, Kidd L. Prospective repeated assessment of self-reported sleep quality and sleep disruptive factors in the intensive care unit: acceptability of daily assessment of sleep quality. BMJ Open 2019;9:e029957. doi:10.1136/ bmjopen-2019-029957

- Prepublication history and additional material for this paper are available online. To view these files, please visit the journal online (http://dx.doi. org/10.1136/bmjopen-2019029957).

Received 21 February 2019

Revised 24 April 2019 Accepted 26 April 2019

D) Check for updates

C Author(s) (or their employer(s)) 2019. Re-use permitted under CC BY-NC. No commercial re-use. See rights and permissions. Published by BMJ.

${ }^{1}$ Nursing, University of Glasgow School of Veterinary Medicine, Glasgow, UK

${ }^{2}$ Nursing, Umm Al-Qura

University, Makkah, Saudi Arabia ${ }^{3}$ University of Glasgow School of Medicine Dentistry and Nursing, Glasgow, UK

${ }^{4}$ School of Medicine Dentistry and Nursing, University of Glasgow, Glasgow, UK

Correspondence to

Ghaida Alsulami;

Gssulami@uqu.edu.sa

\section{ABSTRACT}

Introduction Despite the importance of sleep, the assessment of sleep quality does not form part of standard clinical care in intensive care unit (ICU). Continuous assessment of self-reported quality of ICU patients' sleep has been strongly recommended. Prior to implementing such an assessment in the ICU, it is important to assess the acceptability of this method of assessment to the ICU's patients. The aims of this study were to assess the acceptability to ICU patients of completing daily self-reports on sleep quality during their ICU stay and to assess ICU patients' self-reported sleep quality and sleep disruptive factors during their time in ICU.

Methods An observational prospective-repeated assessment was conducted on $n=120$ patients in an ICU in Saudi Arabia. The participants were both intubated and non-intubated.

Outcomes measures Over a 3-month period, sleep quality was assessed using the Arabic version of the Richards-Campbell Sleep Questionnaire (RCSQ-A), and self-reported sleep disruptive factors were identified. Clinical factors, such as ICU interventions, and previously administered sedatives were also examined. The patients' acceptance of completing daily RCSQ-A reports was assessed using various indicators of acceptability.

Results A total of 381 self-reports (RCSQ-A) were collected for this analysis. The patients reported $34.4 \pm 5.60$, indicating that sleep quality was poor on average. The group of intubated patients reported much poorer sleep quality during intubation than after extubation. In the multivariate analysis, factors which most significantly affected sleep (exp(b), p value) were midazolam $(-6.424, p<0.0005)$, propofol $(-3.600$, $p<0.05)$, noise $(-1.033, p<0.05)$, gender $(1.836, p<0.05)$, daytime sleepiness $(0.856, p<0.05)$ and the presence of mechanical ventilation $(-1.218, p<0.05)$.

Conclusion The acceptability and feasibility of using daily RCSQ-A for sleep quality assessment was demonstrated. Sleep quality was reported as poor by all participants and the factors affecting sleep were varied. This study provided various recommendations for healthcare providers and researchers in terms of evaluating and improving sleep quality in ICU patients.
Strengths and limitations of this study

- This is the first study to assess intensive care unit (ICU) patients' acceptability of completing daily self-reports on the quality of their sleep.

- The prospective repeated-assessment study design facilitated the reduction of recall bias, which allowed sleep disruptive factors to be identified.

- These factors are constantly changing during patients' ICU stays and thus the study design permitted adequate statistical power for analysis.

- The study provided a comparison of self-reported sleep quality during ventilation and after extubation within a group of intubated patients, reducing participant heterogeneity.

- The study was unable to study the effect of the patients' diagnoses and medications on sleep quality due to the variation in their medical conditions and combinations of medication regimes.

\section{BACKGROUND}

Sleep disruption in critically ill patients has been studied for over 30 years. It is defined as a continued lack of restorative sleep over time, resulting in both physical and cognitive impairments. ${ }^{1}$ Studies suggest a number of intrinsic and extrinsic sleep disruptive factors are associated with disrupted sleep in ICU patients. ${ }^{2}$ Extrinsic factors (environmental) include environmental sounds, light and nurses' activities. Intrinsic factors (factors related to the patients) include the severity of the illness, pain, fear or medical treatments. Despite the importance of sleep, continuous assessment of sleep quality during ICU stays does not form part of the standard clinical care given to ICU patients. Assessment is the first critical process in the nursing care plan, which enables nurses to develop an appropriate intervention and evaluate its effectiveness. Regular assessment is important in early 
identification of any sleep problems that ICU patients may have which in turn, lead to identifying the cause of the problem and implementing the proper interventions to improve the patients' sleep. ${ }^{45}$

Sleep assessment in ICUs can be examined using objective tools such as polysomnography (PSG). While PSG is considered the gold standard for sleep measurement, it has certain drawbacks for use in the ICU environment; the electrodes must be worn continuously to collect data on sleep quality and the results require interpretation by experts. ${ }^{67}$ The use of self-report instruments offers an alternative approach to sleep assessment in ICUs. Recent clinical practice guidelines for the management of pain, agitation, delirium and sleep disruption in ICUs, the Society of Critical Care Medicine strongly recommend that patients' sleep should be continuously assessed using a valid assessment tool such as the Richards-Campbell Sleep Questionnaire (RCSQ). ${ }^{8}$ However, prior to implementing self-reports on the quality of patient's sleep as part of the daily routine in the ICU, it is important to determine whether this is acceptable to the patients. Sekhon et al stated that successful implementation of new measures depends on the acceptability of the proposed measures to the recipients. They defined acceptability as a multifaceted construct that reflects the extent to which people participating in new proposed measures consider it to be appropriate, based on their experienced cognitive and emotional responses. A few recent studies have used RCSQ in repeated assessment and throughout the patient's stay in the ICU. ${ }^{10-12}$ They provided data on the completion rate of RCSQ by the participants, ${ }^{10-12}$ and reasons for not completing the RCSQ by some participants. ${ }^{12}$ However, there is no data on the patients' acceptability of this daily assessment (ie, patients' experience of completing RCSQ on a daily basis during their ICU stay).

Despite the growing awareness of the role of sleep in ICU patients' recovery, there is little literature concerning any aspect of ICU patients' sleep quality and sleep disruptive factors in Middle East countries. In particular, the RCSQ is empirically valid and highly recommended for assessing the quality of ICU patients' sleep, there is a lack of knowledge about patients' sleep quality and sleep disruptive factors in Saudi Arabian ICUs, as no study has previously assessed patients' perception of sleep quality and sleep disruptive factors in Saudi Arabian ICUs. Therefore, we adopted a method of prospective repeated assessment of both self-reported sleep quality and sleep disruptive-factors on a daily basis until the patients' discharge from the unit. The primary aims of this study were to: (1) assess the acceptability of ICU patients to complete a daily self-report on sleep quality using the RCSQ during their ICU stay and (2) assess the ICU patients' self-reported sleep quality during their stay in the ICU. The secondary aims were to (3) identify the self-reported factors that disrupt ICU patients' sleep during their ICU stay and (4) evaluate the effects of self-reported sleep disruptive factors and clinical factors on the patients' self-reported sleep quality.
These study aims were designed in the purpose to understand the feasibility of implementing daily self-report assessment of patients' sleep quality in Saudi Arabian ICU clinical practice and its likely performance in national ICU clinical practice and routine care, and to provide a comprehensive view of the quality of ICU patients' sleep and sleep disruptive factors in the Saudi Arabian ICU population. This may help to develop recommendations for healthcare providers and researchers in terms of both examining and improving sleep in ICU patients if necessary.

\section{Methods}

Study design and settings

An observational prospective-repeated assessment was conducted at King Abdul Aziz University Hospital ICU. The hospital is a tertiary referral hospital in the western region of Jeddah, Saudi Arabia. During the study period, the hospital had a total capacity of 845 beds, of which 26 beds were in the ICU facility that provides care for both medical and surgical critically ill patients. A single room was provided for each patient and there was a 1:1 registered nurse-to-patient ratio. The patients' rooms were arranged around a centrally located nursing station.

All rooms featured small windows fitted with blinds. There were no policies in place to schedule the opening and closing of blinds to aid maintenance of circadian rhythm by altering natural light levels according to time of day. Patients' room lights were switched off during the night while the bed lights and corridor lights remained on. There were no set policies or guidelines regarding the patients' sleep quality within the hospital.

\section{Patient involvement}

A group of patients were involved in the design of the study at the planning and pilot testing stages. Those patients provided feedback on the study tools.

\section{Study participants and recruitments}

Participants eligible to take part in the study were adult patients ( $\geq 18$ years) treated in the ICU for $\geq 24$ hours who were alert and interactive, with Glasgow Coma Scale $^{13}$ scores of 15 , including those who were intubated. A convenience sample of all patients who met the eligibility criteria were invited to participate. Exclusion criteria included sedated or agitated patients with Richmond Agitation and Sedation Scale ${ }^{14}$ of $<-1$ or $>+1$, patients with pre-existing sleep pathologies; patients with cognitive dysfunction, and patients who did not speak Arabic. All potential study participants were screened for eligibility at each morning using a study enrolment survey. The participants were assured that participation in the study was not obligatory and were reminded of their right to withdraw at any time.

\section{Data collection}

Data collection was undertaken during May to August 2018. Each participant needed to be alert and calm, therefore, patient consciousness levels were assessed daily; 
similarly, the presence of delirium was assessed using the confusion assessment method for the ICU.

\section{Outcome measures}

Patients' self-report assessment of sleep quality and sleep disturbance factors

The Arabic version of RCSQ (RCSQ-A) ${ }^{15}$ and the modified Sleep in Intensive Care Questionnaire (SICQ) ${ }^{16}$ were administered each morning between 07:00 and 12:00 hours until the day the patient was due to be discharged from the ICU. Details of study instruments can be found in online supplementary material 1 . The five questions from the RCSQ-A were read aloud to the patients and, after each question, the patients rated their previous night sleep by placing a mark on the answer line, which was $100 \mathrm{~mm}$ long $(0 \mathrm{~mm}=$ poorest, $100 \mathrm{~mm}=$ optimum). This approach was chosen to limit the potential of recall bias and to assure optimal reminiscence of the most recent night's sleep. Every attempt was made to ensure that there were no missing or erroneous data by screening each patient's daily assessment sheets. Missing RCSQ-A responses from patients who had declined to complete the questionnaire were not considered and not included in the analyses because the RCSQ total score cannot be computed if the data are incomplete. ${ }^{17}$ The patients who declined to complete the RCSQ-A were asked to clarify whether they were not completing the questionnaire on that individual occasion, or if they were withdrawing from the study. The patients who decided to withdraw from the study were not approached again; however, their consent for data they had already given for analysis was retained. After every RCSQ-A completion, the patients were asked to rate their perception of the factors that disrupted their sleep during the previous night on the modified SICQ scale ( $1=$ no disruption to $10=$ significant disruption). An open-ended question, "What other activities were disruptive to your sleep last night?" was also used. The answers were communicated verbally by most patients and in writing and through actions by some.

\section{Other measures: demographic and clinical data}

Demographic and clinical variables were collected from the patients' medical files. These included age, gender, ICU admission diagnosis, severity of critical illness using the Acute Physiology and Chronic Health Evaluation (APACHE II) score, ${ }^{18}$ length of stay in ICU (ICU-LOS), nightly mechanical ventilation statues (ventilated or non-ventilated) and medications administered during the study. None of the patients were on sedation during the assessment; however, data on previously administered sedation medications were collected.

\section{Patients' acceptance of daily self-reporting on sleep quality using} RCSQ-A during their ICU stays

The key elements of acceptability, including patient willingness, and perceived burden (ability to provide self-reports on a daily basis) were assessed using several indicators of acceptability, ${ }^{9}$ including withdrawal and drop-out rates, the total number of patients who decided to discontinue at some point during the assessment and the total number of completed reports. The participants were also asked: "How did you find completing the questionnaire on sleep quality on multiple days while you were an inpatient in the ICU?"

\section{Sample size}

A post hoc power analysis was conducted using G-Power software V.3.1.9.2 to perform regression analysis for the total sample $n=120$ patients and to determine whether the sample size offered an effective power of at least 0.80 and a significance level of $\alpha=0.05$. This also determined the maximum number of variables to be included in the model. The power analysis revealed that a sample size of 120 was adequate, given the inclusion of 16 variables, with an effective power of 0.88 , assuming a moderate effect size $\left(f^{2}=0.22\right)$. Even supposing a much smaller effect $\left(\mathrm{f}^{2}=0.19\right)$, the power remained 0.82 , which is above the usual minimum requirement of $0.80 .{ }^{19} \mathrm{~A}$ post hoc power analysis was also used to perform a paired samples t-test for the subsample of $n=43$ patients who were placed on ventilation during the study assessment. The analysis revealed that on the basis of the mean, between two different conditions (during ventilation and after extubation), a sample size of 43 patients was adequate to obtain a statistical power of 0.89 with an effect size $(d=0.5)$ and a significance level of $\alpha=0.05$.

\section{Data management and analysis}

The data were analysed using IBM-SPSS V.23.0. The data were first assessed for normality using the Shapiro-Wilk test. ${ }^{20}$ Descriptive statistics including frequencies and percentages were used to describe the categorical data, for example, gender, admission diagnosis. The mean, range and SD were used to describe the continuous data for the total sample of $n=120$ patients' sleep quality and sleep disruptive factors. Descriptive statistics were also used to describe a subsample of $n=43$ patients who had been placed on a ventilator at some point during the study period. The description included their self-reported sleep quality and sleep disruptive factors when they were on the ventilation and after they were extubated. A paired sample t-test was performed to determine whether there was a significant difference in patients' sleep quality during ventilation and after extubation, and whether there were significant differences in self-reported sleep disruptive factors during ventilation and after extubation that were explained by intubation status. The two-sided statistical significance level was set to $0.05 \%$ and $95 \%$ CIs were used.

The total RCSQ-A score for the total sample $n=120$ was converted into an estimate of the Sleep Efficiency Index (SEI) using the following formula:

SEI $=46.88+(0.39 \times$ RCSQ $){ }^{10} 1721$

The correlation between daytime sleepiness and ICU-LOS was assessed using a bivariate (Pearson's) correlation. A multiple regression was conducted for the total 
sample to assess the significance and relative contribution of each independent variable on predicting the dependent variable sleep quality. To avoid overfitting the model, only the factors rated most highly by the patients were included in the model in addition to the variables of interest, reflecting the demographic and clinical characteristics derived from previous research. The appropriate modelling of continuous variables was confirmed by evaluating their linearity. The intercorrelation between independent variables for this repeated assessment was assessed using variance inflation factor (VIF) values $<10$, and tolerance values $>0.1$. It was addressed by re-categorising the relevant collinear variables. The majority of patients $(87.5 \%)$ in this study received non-opioid analgesics alongside opioid-fentanyl analgesics. To avoid multicollinearity, these two variables were combined into a single variable and entered as 'analgesic'. The independence of observation (residuals) was assessed by DurbinWatson statistic values of 1.299. Content analysis was used to interpret and synthesise the data collected in the openended questions. ${ }^{22}$

\section{RESULTS}

A total of 354 patients were screened, of whom 224 were excluded because they did not meet the inclusion criteria (figure 1). The remaining 130 patients met the inclusion criteria, however, 10 patients were unwilling to participate in any research studies and declined to participate, leaving 120 patients enrolled. The average age of the patients was 59 years, and the majority (60\%) were male. The participants' APACHE II score within 24 hours of ICU admission ranged from 10 to 24 with an average of $15.78 \pm 2.606$. More than half the participants had an APACHE II score between 10 and $16 \mathrm{n}=71$ (59.2\%); meanwhile, 49 participants $(40.8 \%)$ had a higher score between 17 and 24 . The average ICU-LOS was 9.35 days \pm 3.15 . Patients who had an APACHE II score between 10 and 16 stayed in the ICU for 4-12 days. In addition, patients who had a score between 17 and 24 stayed in the ICU for 6-21 days. Of the study sample, $43(35.8 \%)$ patients were on mechanical ventilation (MV). Table 1 provides a summary of the sample demographic and treatment characteristics during the study assessment.

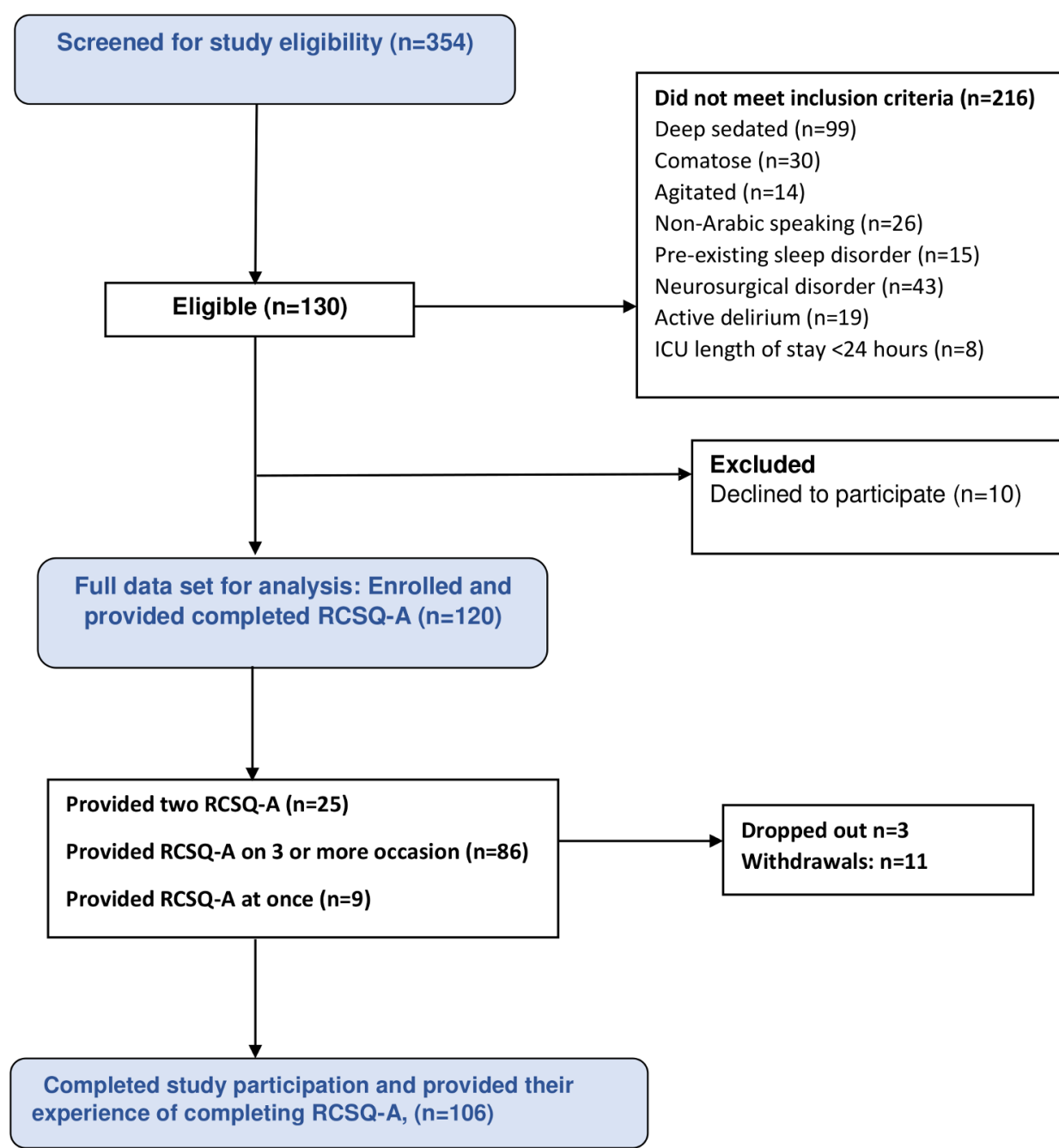

Figure 1 Flow diagram of patients' screening, enrolment and participants' RCSQ-A completion. ICU, intensive care unit; RCSQ-A, Arabic version of the Richards- Campbell Sleep Questionnaire. 
Table 1 Demographic and clinical characteristics of patients $(n=120)$

\begin{tabular}{|c|c|c|c|}
\hline Characteristics & Category & n (\%) & Range \\
\hline Age $($ mean $\pm S D)$ & $59.7 \pm 9.44$ & & $19.00-75.00$ \\
\hline Gender & $\begin{array}{l}\text { Male } \\
\text { Female }\end{array}$ & $\begin{array}{l}72(60) \\
48(40)\end{array}$ & \\
\hline \multirow[t]{8}{*}{ Admission diagnosis } & Medical cardiac & $21(17.5)$ & \\
\hline & Medical respiratory & $21(17.5)$ & \\
\hline & Gastrointestinal & $11(9.1)$ & \\
\hline & Other & $8(6.7)$ & \\
\hline & Surgical postoperative & $59(49.2)$ & \\
\hline & Cardiothoracic & $37(30.9)$ & \\
\hline & Thoracic traumatic & $12(10)$ & \\
\hline & Abdominal & $10(8.3)$ & \\
\hline APACHE II score (mean \pm SD) & $\begin{array}{l}15.78 \pm 2.606 \\
\text { Low } \\
\text { Medium }\end{array}$ & $\begin{array}{l}71(59.2) \\
49(40.8)\end{array}$ & $\begin{array}{l}10.00-24.00 \\
10.00-16.00 \\
17.00-24.00\end{array}$ \\
\hline Length of ICU stay (mean \pm SD) & $9.35 \pm 3.15$ & & $4.00-21.00$ \\
\hline Medications ${ }^{*}$ & $\begin{array}{l}\text { Beta-blockers } \\
\text { Diuretics } \\
\text { Calcium channel blockers } \\
\text { Corticosteroids } \\
\text { Adrenergic } \\
\text { Non-opioid and opioid } \\
\text { Non-opioid paracetamol }\end{array}$ & $\begin{array}{l}75(62.5) \\
76(63.5) \\
99(82.5) \\
45(37.5) \\
39(32.5) \\
105(87.5) \\
15(12.5)\end{array}$ & \\
\hline Sedation & $\begin{array}{l}\text { Propofol } \\
\text { Benzodiazepines (midazolam) } \\
\text { Dexmedetomidine (Precedx) }\end{array}$ & $\begin{array}{l}54(45) \\
40(33.3) \\
26(21.7)\end{array}$ & \\
\hline $\begin{array}{l}\text { Richmond Agitation and Sedation Scale } \\
\text { on enrolment }\end{array}$ & Alert and calm (zero score) & $120(100)$ & \\
\hline Glasgow Coma Scale & Fully conscious (15 score) & $120(100)$ & \\
\hline Developed delirium & $\begin{array}{l}\text { Positive confusion assessment method for } \\
\text { the ICU }\end{array}$ & $11(9.2)$ & \\
\hline Intubation statues & Intubated & $43(35.8)$ & \\
\hline Method of ventilation & $\begin{array}{l}\text { Invasive ventilation } † \\
\text { Non-invasive }\end{array}$ & $\begin{array}{l}30(69.8) \\
13(30.2)\end{array}$ & \\
\hline $\begin{array}{l}\text { Duration of mechanical ventilation } \\
(\text { mean } \pm S D)\end{array}$ & $6.26 \pm 3.381$ & & $2.00-17.00$ \\
\hline
\end{tabular}

*Beta-blocker=metoprolol, carvedilol; diuretics=metolazone, furosemide, amiloride; calcium channel blockers=amlodipine, verapamil; corticosteroids=prednisolone, dexamethasone, hydrocortisone; adrenergic=norepinephrine, epinephrine or dopamine.

†Ventilation applied via tracheotomy or endotracheal.

¥Ventilation applied via face or nasal mask.

APACHE, Acute Physiology and Chronic Health Evaluation; ICU, intensive care unit.

\section{Participants' self-reported assessments of sleep and sleep disruptive factors}

The average sleep quality as reported by the patients was poor, with mean scores for each of the RCSQ-A elements below $50 \mathrm{~mm}$ (table 2). Furthermore, the mean SEI was $60.3 \%$, and an SEI $<85 \%$ indicates poor sleep quality. ${ }^{17}$ In contrast, the average self-reported sleep quality at home was described as good, with a mean score of $7.16 \pm 1.754$. The average daytime sleepiness score was $5.52 \pm 1.52$, and daytime sleepiness did not change significantly over the course of any patient's ICU stay $(p>0.05)$. Multiple factors were reported to disrupt patients' sleep (table 3). Patients rated noise as the most disruptive extrinsic factor at $7.48 \pm 1.57$, followed by clinical interventions at $5.95 \pm 1.57$; the highest rated noise was talking at $6.80 \pm 1.25$, while the highest rated intrinsic disruptive factor was fear at 3.64 \pm 2.01 . Online supplementary material 2 shows patients' comments on other factors that disrupted their sleep, including the categories and the subcategories that emerged from content analysis in accordance with the study by Edéll-Gustafsson $e t a .^{23}$ 


\begin{tabular}{|c|c|c|}
\hline RCSQ-A items & Mean \pm SD & Range \\
\hline (RCSQ-A.1) Sleep depth & $31.82 \pm 7.03$ & $19-56$ \\
\hline (RCSQ-A.2) Falling asleep & $33.07 \pm 6.73$ & $21-54$ \\
\hline (RCSQ-A.3) Awakenings & $35.06 \pm 5.76$ & $18-47$ \\
\hline (RCSQ-A.4) Returning to sleep & $36.29 \pm 5.36$ & $25-50$ \\
\hline (RCSQ-A.5) Overall sleep quality & $35.36 \pm 5.34$ & $22-51$ \\
\hline Total RCSQ-A score* & $34.41 \pm 5.60$ & $23-48$ \\
\hline SEl† & 60.30 & \\
\hline
\end{tabular}

*Total RCSQ-A=average of five items (Q1-Q5). The total RCSQ-A score was categorised, with a cut-off point of $<26$ indicating very poor sleep quality, a score of (26-50) indicating poor sleep quality, a score of (51-75) indicating good sleep quality and a score of $>75$ indicating very good sleep quality. ${ }^{29} 30$

$\dagger S E I \leq 85 \%$ indicates poor sleep quality.

RCSQ-A, Arabic version of the Richards-Campbell Sleep

Questionnaire; SEI, Sleep Efficiency Index.

\section{Factors affecting sleep quality}

The predictor variables included in the multiple regression model were noise, clinical interventions, talking, machine alarms and fear, in addition to the intrinsic factors of age, gender, daytime sleepiness, APACHE II score, ICU-LOS, analgesic use, MV status and previous receipt of sedation using midazolam, propofol and Precedx. The full model (table 4) explained $39.3 \%$ of the variance in total sleep quality, a statistically significant predictor of total sleep quality, with the $R^{2}=0.423$, $F(6.113)=13.828$ and $p<0.0005$. The factors which negatively and significantly affected sleep quality (given as $(\exp (\mathrm{b})(95 \% \mathrm{CI}), \mathrm{p}$ value $))$ were midazolam $(-6.424$ (-8.99 to -3.86$), \mathrm{p}<0.0005)$, propofol $(-3.600$ ( -5.71 to $-1.49), \mathrm{p}<0.05)$, noise $(-1.033(-1.70$ to -0.364$), \mathrm{p}<0.05)$ and the presence of a ventilator $(-1.218(-2.36$ to -0.077$)$, $\mathrm{p}<0.05)$. Total sleep quality was also significantly affected

Table 3 Self-reported sleep disruptive factors on modified Sleep in Intensive Care Questionnaire $(n=120)$

\begin{tabular}{|c|c|c|}
\hline $\begin{array}{l}\text { Sleep disruptive factors in } \\
\text { rank order }\end{array}$ & Mean \pm SD & Range \\
\hline Noise & $7.48 \pm 1.57$ & $3.00-9.00$ \\
\hline $\begin{array}{l}\text { Clinical interventions (ie, } \\
\text { blood samples, vital signs, } \\
\text { etc) }\end{array}$ & M5.95 \pm 1.86 & $2.30-9.00$ \\
\hline Light & $2.36 \pm 0.94$ & $1.00-5.00$ \\
\hline Talking & $6.80 \pm 1.25$ & $1.00-9.00$ \\
\hline $\begin{array}{l}\text { Machine alarms (ie, heart } \\
\text { monitor, ventilator, etc) }\end{array}$ & $4.31 \pm 2.35$ & $1.00-9.00$ \\
\hline Telephone & $1.12 \pm 0.36$ & $1.00-7.30$ \\
\hline Fear & $3.64 \pm 2.01$ & $1.00-8.25$ \\
\hline Pain & $2.30 \pm 1.10$ & $1.00-7.30$ \\
\hline $\begin{array}{l}\text { Discomfort of being attached } \\
\text { to the devices }\end{array}$ & $2.26 \pm 1.18$ & $1.00-5.75$ \\
\hline
\end{tabular}

by differences in gender such that predicted sleep quality for female patients was greater than for male patients (1.836 (0.157 to 3.52), $\mathrm{p}<0.05)$. Furthermore, daytime sleepiness significantly affected the patients' sleep: using a daytime sleepiness scale, where $1=$ unable to stay awake and $10=$ fullyalert and awake, any increase on the scale was associated with a significant increase in total sleep quality (0.856 (0.175 to 1.54$), \mathrm{p}<0.05)$.

\section{Self-reported sleep quality and sleep disruptive factors reported by participants during intubation and after extubation}

The subsample of 43 patients who were placed on MV during the study reported sleep quality during intubation $(31.88 \pm 6.16)$ as much poorer than after extubation (35.04 \pm 6.47$)$; these differences were significant with $\mathrm{p}<0.0005$. Patients reported sleep fragmentation as the greatest disturbance during intubation (30.63 \pm 5.79$)$. Following extubation, the number of awakenings was significantly reduced, to a mean of $36.81 \pm 6.83$ (table 5). There were significant differences between the level of reporting for several sleep disruptive factors during ventilation and after extubation ( $\mathrm{p}<0.05)$, as shown in figure 2 . During ventilation, machine alarms, clinical intervention and fear were rated as causing high levels of sleep disruption $(7.19 \pm 1.13,7.04 \pm 2.04$ and $6.32 \pm 1.81$, respectively). However, following extubation, these levels of disruption reduced significantly, causing only moderate to mild levels of disruption $(4.68 \pm 1.37,6.07 \pm 2.34$ and $2.72 \pm 1.34$, respectively).

\section{Patients' acceptance of making daily self-reports on sleep quality using RCSQ-A during ICU stays}

Dropout and withdrawal rates

The number of patients who dropped out by choosing to stop participating was very small at $\mathrm{n}=3(2.5 \%)$. No reasons for such cessations were provided. The number of withdrawals was also very small at $\mathrm{n}=11(9.2 \%)$. These patients were withdrawn because they no longer met the study's inclusion criteria, as they had become agitated and developed delirium. The majority of participants, $\mathrm{n}=106(88.4 \%)$, were able to complete study participation in full.

\section{Number of completed self-reports (RCSQ-A)}

In total, 381 reports were collected from 120 participants. The answers to the open-ended question confirmed that most participants, $\mathrm{n}=89(83.9 \%)$, were happy to complete the RCSQ-A daily during their stay in the ICU. However, some of the participants, $\mathrm{n}=17(16.1 \%)$, at some point during the repeated assessment did not complete the daily RCSQ-A; these patients had some difficulties in completing the questionnaire for personal reasons such as feeling tired or bored (online supplementary material $3)$.

\section{Experiences of completing the RCSQ-A}

The time taken to complete RCSQ-A was between 2 and 3 min. The participants completed the RCSQ-A between 
Table 4 Model summary of the stepwise multiple regressions predicting total sleep quality from sleep disruptive factors with (adjusted $\mathrm{R}^{2}=0.393$ )

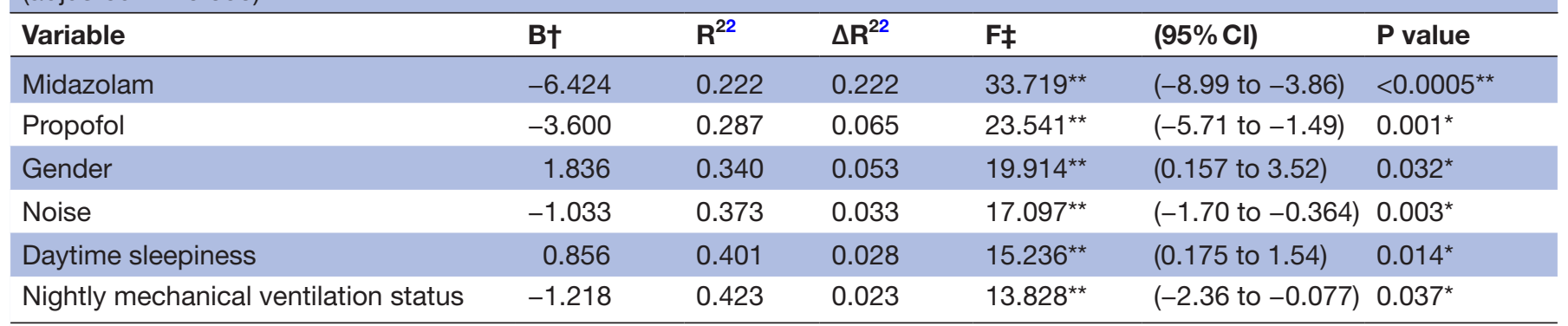

${ }^{*} \mathrm{P}<0.05 ;{ }^{* *} \mathrm{P}<0.0005$ is highly significant.

$\dagger B$, unstandardised regression coefficients.

$\dagger F$, test of overall significance.

one and six times, with the average being three times. In total, 111 patients $(92.5 \%)$ provided more than one RCSQ-A, while only 9 participants $(7.5 \%)$ provided a single self-report. Four of the participants became delirious and agitated on the second day of assessment, while three patients asked to stop participating; two patients were discharged from the ICU on their second day of assessment. Among the study participants, 68 (56.7\%) were unable to set a mark on the Visual Analogue Scale (VAS) themselves, requiring assistance due to physical barriers such as hand tremors and muscle weakness. These patients were only able to point at their chosen spots on the scales.

\section{DISCUSSION}

This study was designed to assess the acceptability of ICU patients' completion of daily self-reports (RCSQ-A) on their sleep quality throughout their ICU stay and to assess self-reported sleep quality and sleep disruptive factors on a daily basis until patients were discharged from the unit. It is important to study sleep quality and sleep disruptive factors simultaneously to develop a comprehensive picture of the patients' sleep quality and the factors that disrupt it. We considered that this would inform the future development of strategies to improve patients' sleep in the ICU. A review of the literature suggests that this is the first study on ICU patients' experience of completing daily self-reports on their sleep quality during their ICU stay. It is also the first study that has assessed self-reported sleep quality using a valid tool (RCSQ-A) and self-reported sleep disruptive factors in ICU patients in an Arabic-speaking country in the Middle East.

There was evidence of general poor sleep quality in this cohort of ICU patients. The overall quality of sleep from the patient perspective was 34.41 , which is lower than the reported findings in previous studies. ${ }^{10}{ }^{124-28}$ The SEIs emerged at $60.3 \%$, matching the results from a group of ICU patients in the $\mathrm{UK}^{27}$ used as a control, in which the SEIs were $60.8 \%$, and slightly lower than reported in the repeated self-report assessment study from Australia $(60.3 \%$ vs $65 \%) .{ }^{10}$ In this study, patient perception of sleep varied from poor to very poor in contrast to other self-report assessment studies in which patients' sleep varied from very good to very poor. ${ }^{12}{ }^{24} 28-30$ These differences may be due to the different treatment characteristics of the patients, as this study included intubated patients, and the different ICU environment. Differences in the method of sleep quality assessment could also have

Table 5 Self-reported sleep quality of patients when they were intubated and after extubation $(n=43)$

\begin{tabular}{|c|c|c|c|c|c|}
\hline \multirow[b]{2}{*}{ RCSQ-A items } & \multicolumn{2}{|c|}{ Patients on ventilation $n=(43)$} & \multicolumn{2}{|c|}{ Patients after extubation $n=(43)$} & \multirow[b]{2}{*}{$\mathrm{P}$ value† } \\
\hline & Mean \pm SD & Range & Mean \pm SD & Range & \\
\hline Depth of sleep (RCSQ-A.1) & $32.00 \pm 9.13$ & $21-53$ & $33.43 \pm 8.58$ & $18-51$ & $0.001^{*}$ \\
\hline Falling asleep (RCSQ-A.2) & $33.00 \pm 8.67$ & $19-53$ & $34.38 \pm 8.41$ & $22-56$ & $0.001^{*}$ \\
\hline Number of awakenings (RCSQ-A.3) & $30.63 \pm 5.79$ & $15-41$ & $36.81 \pm 6.83$ & $19-56$ & $<0.0005^{\star \star}$ \\
\hline Returning to sleep (RCSQ-A.4) & $31.85 \pm 5.50$ & $21-40$ & $36.20 \pm 5.99$ & $28-49$ & $<0.0005^{\star \star}$ \\
\hline Overall sleep quality (RCSQ-A.5) & $32.14 \pm 5.51$ & $21-41$ & $34.40 \pm 5.54$ & $25-47$ & $<0.0005^{\star \star}$ \\
\hline Overall (RCSQ-A) scoreł & $31.88 \pm 6.16$ & $20-45$ & $35.04 \pm 6.47$ & $24-49$ & $<0.0005$ \\
\hline
\end{tabular}

${ }^{*} \mathrm{P}<0.05,{ }^{* *} \mathrm{P}<0.0005$ is highly significant.

†Paired t-test.

$\ddagger$ Average of 5 items (Q1-Q5).

RCSQ-A, Arabic version of the Richards-Campbell Sleep Questionnaire. 


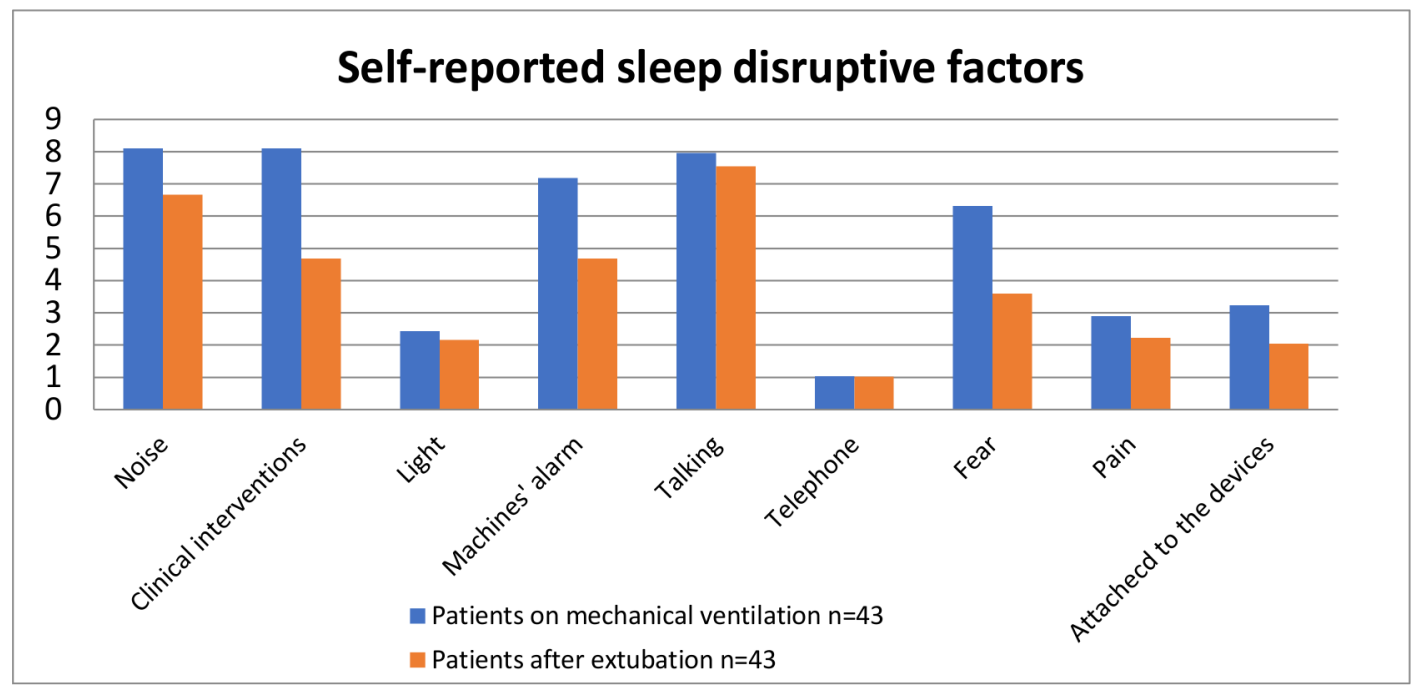

Figure 2 Significance of changes in the self-reported sleep disruptive factors during intubation and after extubation.

influenced these results. The current results are based on continuous assessment until discharge from the ICU, while the majority of previous studies limited assessment to a single night. ${ }^{362124}$ Only three previous studies used RCSQ for repeated assessment, ${ }^{10-12}$ and their assessments were limited to non-intubated patients. The finding that patients' sleep is reported as worse in the ICU than at home is consistent with previous studies. ${ }^{361621}$ This indicates that there are factors within these environments which may lead to changes in and disruption to patients' sleep.

The results demonstrated that daytime sleepiness was consistent with lack of sleep during the night and that perceived daytime sleepiness did not improve over the course of patients' ICU stay. These results are consistent with previous polysomnographic and self-report studies, ${ }^{3}{ }^{31}$ which showed that between $40 \%$ and $50 \%$ of total sleep time in an ICU occurs during the day, and that this altered sleep pattern did not improve over the course of the stay. It is known that female subjects experience additional slow wave sleep, and this is reflected by the observed gender differences in patients' sleep in this study: female patients slept better than male patients. This supports a recent study, which found that female patients had better sleep than male. ${ }^{3}$ Our results showed that multiple sleep disrupting factors were identified by the entire sample, which substantiates other results. Of the extrinsic factors, patients rated noise as the most disruptive, supporting the findings of previous studies. ${ }^{3} 163233$ Peak noise levels in the ICU were documented at 41 and $68 \mathrm{~dB},{ }^{33-35}$ exceeding the WHO's recommendation for sound levels in an ICU not to exceed $35 \mathrm{~dB}$ during the day and $30 \mathrm{~dB}$ at night. ${ }^{36}$ The results also support the idea that interruptions of sleep in the ICU caused by clinical interventions are important. This finding is consistent with the results of Celik $e t a l,{ }^{37}$ who found that patients had their sleep interrupted by human interventions an average of 51 times each per night. However, in addition to these, psychological factors cannot be ignored. Patients reported fear to be the most disruptive intrinsic factor; they also referred to nightmares and worries, corroborating previous study findings. 102930

The effects of sedation in the ICU have not been studied sufficiently. None of the patients were on sedation during this assessment, however, data on previously administered sedation were gathered. Sedatives, especially benzodiazepines, are commonly used to induce sleep, however, these have been known to suppress slow wave sleep (SWS) and rapid eye movement sleep (REM) after withdrawal. ${ }^{38}$ Propofol has shown to increase SWS while suppressing REM sleep. ${ }^{31}$ Interestingly, patients who received benzodiazepines had worse sleep quality than patients who received propofol. However, both forms of sedation significantly affected patients' sleep quality. The adverse effects of many sedatives have been well documented, and thus sedatives should not be used for sleep promotion in most $\operatorname{cases}^{8}$; in addition, patients receiving these drugs should be carefully monitored with regard to the quality of their sleep.

The negative effects of the presence of MV on patients' sleep quality have been reported in several polysomnographic studies. ${ }^{313940}$ However, this is the only self-report study that included intubated patients and assessed their perception of sleep quality alongside their perceptions of sleep disruptive factors on a daily basis both during intubation and after extubation to determine whether the ventilator has an effect on the patients' perception of sleep and the factors that disrupt their sleep. Intubated patients reported better sleep quality following extubation and the differences were statistically significant. However, to date there is no information which provides guidance about clinically important changes in the RCSQ scores, and thus it is difficult to make too much of the result. The patients also reported sleep fragmentation to be greater during intubation than after extubation. Furthermore, during ventilation, the factors of machine alarms, clinical interventions and fear were rated by the patients as the most disruptive factors, while after extubation, the level of 
disruption reduced significantly. One possible explanation for high sleep fragmentation during intubation is the disruptive factors that arise from or are increased by the presence of the ventilator, such as alarms, clinical interventions and feelings of fear. Freedman $e t a b^{31}$ assessed the sleep quality of ventilated patients and demonstrated that sleep was highly fragmented; they suggested that this may be due to the multiple human interventions during ventilation. Our findings stress the need for attention to be paid to the sleep quality of this group of patients, with close monitoring for factors that may adversely affect sleep. In particular, environmental factors such as noise from alarms should not be overlooked. Such impacts should be handled properly by following guidelines such as the Joint Commission International policies on safely managing clinical alarm systems to avoid false alarms. ${ }^{41}$ Clustering patients' care activities as much as possible and avoiding performing unnecessary care activities during the night is also important for managing these factors. Where MV is present, the patients may experience distressing psychological side effects such as fear, ${ }^{42}$ therefore, it is important to consider the individual patient's psychological needs.

Our results demonstrated that daily self-report assessments on sleep quality using the RCSQ-A was non-burdensome to the majority of participants. Therefore, it is somewhat surprising that the use of RCSQ for repeated assessment in ICUs is only infrequently published, with only three main studies of this type. ${ }^{10-12}$ Two were conducted in Australia, ${ }^{10} 12$ with one featuring 151 participants reporting on their sleep using the RCSQ 356 times where $50 \%$ of the participants were able to report on 2 or more days ${ }^{10}$; the other Australian study ${ }^{12}$ featured 50 patients reporting, and the completion rate was $72 \%$. The third study, in North America, ${ }^{11}$ featured 33 patients over 137 days completing 121 self-reports, giving a rate of $88 \%$. These studies and our own completion rate of $92.5 \%$ provide evidence to support the tool's feasibility for routine assessment of patients' sleep in ICUs. The patients in this study generally described their experience of completing the RCSQ-A during their stays positively. The patients were happy and reported that various psychological and social needs were met by this method of assessment. For example, they felt a sense of security, enhanced communication levels, reduced feelings of loneliness and a sense that someone respected and cared about their need for sleep. These results support a recent qualitative study, ${ }^{44}$ which found that patients who felt well taken care of felt more relaxed and reported that their sleep was positively affected.

According to the daily self-report assessment of both sleep quality and sleep disruptive factors, sleep disruptive factors were evaluated differently from day to day and patient to patient despite the constant setting. Therefore, the findings do not support the hypothesis that ICU noise is the main factor responsible for sleep disturbance for all ICU patients. ${ }^{45}$ Patients' sleep disruption is influenced by several inter-related factors that constantly change due to the nature of the ICU environment. Sensitivity to sources of sleep disruption also varies from patient to patient. ${ }^{31}$ Unfortunately, individual differences were not considered in most recent intervention studies that aimed to improve sleep in the ICU by developing and applying protocols. ${ }^{21} 252728$ Recent guidelines ${ }^{8}$ also revealed problems with the methodology in these intervention studies, highlighting the need for well-designed non-pharmacological measures and improved methods for measuring sleep to allow the implementation of interventions with individualised approaches.

Our findings demonstrate the acceptability and feasibility of using repeated, self-reported RCSQ assessments of sleep quality in ICU environments. Such assessments can be performed whenever the patients are sufficiently alert, and they do not need to be able to communicate verbally. The findings also encourage clinicians routinely inquire about patients' sleep and, implement routine early documentation of sleep patterns using RCSQ in the patient care plans. Patient perceptions of the factors disrupting sleep should be identified individually to determine the patient-specific needs to address sleep disturbances with treatment decisions. Furthermore, patients should be involved in their care; this corresponds with the Institute for Healthcare Improvement's identification of patient safety as one driver of exceptional patient-centred care. ${ }^{46}$ However, it would be valuable to further validate the RCSQ in intubated ICU populations, as the original validation was performed using PSG in non-intubated patients. ${ }^{17}$ This additional validation would enhance the promotion and use of this instrument for the purposes of ongoing assessment over various points of the patients' ICU stays. Further studies are required to test acceptance in other populations of ICU patients in different countries and regions. Further work is also required to assess the perceptions and acceptance of healthcare providers in ICUs in terms of implementation. The quality of sleep was poor in all participants in this case, highlighting the need for further testing in Middle Eastern countries. The facilitation of high-quality sleep for ICU patients is often overlooked by healthcare professionals. However, the results of this study suggest that better education should be provided regarding the negative effects of poor sleep for patients, and training should be established to allow healthcare providers to mitigate these effects. Additionally, to ensure high standards of care in the ICU, hospitals should introduce policies to avert sleep disturbances and should regularly assess the sleep quality of patients, aiming to allow patients sufficient rest periods of a minimum of $90 \mathrm{~min}$ to experience a full sleep cycle. To meet these aims, individual patient planning may be required.

This study had several limitations, which must be acknowledged. Selection bias is possible, as all patients selected to participate were non-sedated; this was necessary, as sedation affect cognitive abilities, and, therefore, would affect the validity of the results. However, this means that the results are not generalisable to the 
whole ICU patient population. Nevertheless, this is an important patient population to study, especially as it includes patients in the period after sedation cessation, when regular assessment of sleep quality that may be affected by the previously received sedation is necessary. The other issue with the findings is generalisability; half of the study sample were postoperative surgical ICU patients and most of them were cardiac patients. This limits the study's findings to these patients. Future research will be required in a broader critical care population. The aim was to assess sleep quality subjectively from a patient perspective, consequently, changes in sleep architecture were not observable, due to the use of a self-report tool. However, routine use of objective methods of assessment such as PSG monitors during patient care is not feasible, and the clinically meaningful outcome of sleep quality is the patient's experience. ${ }^{812}$

\section{CONCLUSION}

Sleep quality was reported as poor by all participants. The factors affecting sleep were multiple and varied from patient to patient, stressing the need to regularly and individually assess patients' sleep quality, and the importance of adopting patient-centred care, including an individual sleep care plan for each patient. The results also demonstrated the feasibility and the acceptability of ICU patients completing daily self-reports of their sleep quality using the RCSQ-A during their ICU stays.

Acknowledgements The authors would like to thank Saudi Cultural Bureau in London, UK and Umm Al-Qura University for sponsoring this study and University of Glasgow for valuable support. The authors would like to thank the head nurse of the ICU Mrs. Shehla Nuzhat, and Mrs. Abeer Al-Hazmi (Assistance nurse director-education and development) at King Abdul Aziz University Hospital for their contribution in facilitating the process for data collection and the patients for their generous contribution to the study. The authors would like to thank Dr Anna 0'Neill and Dr Joanne McPeake at University of Glasgow who provided insight and expertise that assisted the research. The authors would also like to thank Professor Siraj Wali, Professor Faris Al-Hejaili (Consultant in Respiratory and Sleep Medicine) and Dr Ali Al-Faidh (Consultant in Intensive Care Unit) at King Abdul Aziz University Hospital for their review of the study instruments and for facilitating data collection process.

Contributors All authors were responsible and accountable to all part of works related to the study. More specifically, GA had the original idea. AMR and LK contributed to the conception and design of the study. GA collected data and analysed the data. AMR and LK contributed to the interpretation of data. AMR and LK contributed in writing the manuscript. All authors revised the manuscript and gave the approval to the final version to be published.

Funding This study was supported by grants from Saudi Cultural Bureau in London, UK and Umm Al-Qura University, Saudi Arabia.

Disclaimer The funder has no role in study design or the collection, management, analysis or interpretation of data.

Competing interests None declared.

Patient consent for publication Not required.

Ethics approval The study was reviewed and approved by the Institutional Review Board, King Abdul Aziz University Hospital, Saudi Arabia (ref number: 612-17), and the University of Glasgow, UK (ref number: 200170066).

Provenance and peer review Not commissioned; externally peer reviewed.

Data sharing statement All data relevant to the study are included in the article or uploaded as supplementary information.
Open access This is an open access article distributed in accordance with the Creative Commons Attribution Non Commercial (CC BY-NC 4.0) license, which permits others to distribute, remix, adapt, build upon this work non-commercially, and license their derivative works on different terms, provided the original work is properly cited, appropriate credit is given, any changes made indicated, and the use is non-commercial. See: http://creativecommons.org/licenses/by-nc/4.0/.

\section{REFERENCES}

1. Parthasarathy S, Tobin MJ. Sleep in the intensive care unit. Intensive Care Med 2004;30:197-206.

2. Elliott R, Rai T, McKinley S. Factors affecting sleep in the critically ill: an observational study. J Crit Care 2014;29:859-63.

3. Bihari S, Doug McEvoy R, Matheson E, et al. Factors affecting sleep quality of patients in intensive care unit. J Clin Sleep Med 2012;8:301-7.

4. Jeffs EL, Darbyshire JL. Measuring sleep in the intensive care unit: a critical appraisal of the use of subjective methods. J Intensive Care Med 2017:088506661771219.

5. Hoey LM, Fulbrook P, Douglas JA. Sleep assessment of hospitalised patients: a literature review. Int J Nurs Stud 2014;51:1281-8.

6. Elliott R, McKinley S, Cistulli P, et al. Characterisation of sleep in intensive care using 24-hour polysomnography: an observational study. Crit Care 2013;17:R46.

7. Knauert MP, Yaggi HK, Redeker NS, et al. Feasibility study of unattended polysomnography in medical intensive care unit patients. Heart Lung 2014:43:445-52.

8. Devlin JW, Skrobik Y, Gélinas C, et al. Clinical Practice Guidelines for the Prevention and Management of Pain, Agitation/Sedation, Delirium, Immobility, and Sleep Disruption in Adult Patients in the ICU. Crit Care Med 2018;46:e825-e873.

9. Sekhon M, Cartwright M, Francis JJ. Acceptability of healthcare interventions: an overview of reviews and development of a theoretical framework. BMC Health Serv Res 2017;17:88.

10. Aitken LM, Elliott R, Mitchell M, et al. Sleep assessment by patients and nurses in the intensive care: An exploratory descriptive study. Aust Crit Care 2017;30:59-66.

11. Kamdar BB, Shah PA, King LM, et al. Patient-nurse interrater reliability and agreement of the Richards-Campbell sleep questionnaire. Am J Crit Care 2012;21:261-9.

12. Menear A, Elliott R, M Aitken L, et al. Repeated sleep-quality assessment and use of sleep-promoting interventions in ICU. Nurs Crit Care 2017;22:348-54.

13. Fischer M, Rüegg S, Czaplinski A, et al. Inter-rater reliability of the Full Outline of UnResponsiveness score and the Glasgow Coma Scale in critically ill patients: a prospective observational study. Crit Care 2010;14:R64.

14. Sessler CN, Gosnell MS, Grap MJ, et al. The Richmond AgitationSedation Scale. Am J Respir Crit Care Med 2002;166:1338-44.

15. Al-Sulami G, Rice AM, Kidd L, et al. An Arabic translation, reliability, validity and feasibility of the Richards-Campbell Sleep Questionnaire for Sleep Quality Assessment in Intensive Care Unit: ProspectiveRepeated Assessments. J Nurs Meas. forthcoming.

16. Freedman NS, Kotzer N, Schwab RJ. Patient perception of sleep quality and etiology of sleep disruption in the intensive care unit. $A m$ $J$ Respir Crit Care Med 1999;159:1155-62.

17. Richards KC, O'Sullivan PS, Phillips RL. Measurement of sleep in critically ill patients. J Nurs Meas 2000;8:131-44.

18. Knaus WA, Draper EA, Wagner DP, et al. APACHE II: a severity of disease classification system. Crit Care Med 1985;13:818-29.

19. Cohen J. A power primer. Psychol Bull 1992;112:155-9.

20. Shapiro SS, Wilk MB. An analysis of variance test for normality (complete samples). Biometrika 1965;52:591-611.

21. Li SY, Wang TJ, Vivienne Wu SF, et al. Efficacy of controlling nighttime noise and activities to improve patients' sleep quality in a surgical intensive care unit. J Clin Nurs 2011;20:396-407.

22. Vaismoradi M, Turunen $\mathrm{H}$, Bondas T. Content analysis and thematic analysis: Implications for conducting a qualitative descriptive study. Nurs Health Sci 2013;15:398-405.

23. Edéll-Gustafsson U, Arén $\mathrm{C}$, Hamrin E, et al. Nurses' notes on sleep patterns in patients undergoing coronary artery bypass surgery: a retrospective evaluation of patient records. J Adv Nurs 1994;20:331-6.

24. Nicolás A, Aizpitarte E, Iruarrizaga A, et al. Perception of night-time sleep by surgical patients in an intensive care unit. Nurs Crit Care 2008;13:25-33.

25. Kamdar BB, King LM, Collop NA, et al. The effect of a quality improvement intervention on perceived sleep quality and cognition in a medical ICU. Crit Care Med 2013;41:800-9. 
26. Ritmala-Castren M, Axelin A, Kiljunen K, et al. Sleep in the intensive care unit - nurses' documentation and patients' perspectives. Nurs Crit Care 2017;22:238-46.

27. Patel J, Baldwin J, Bunting P, et al. The effect of a multicomponent multidisciplinary bundle of interventions on sleep and delirium in medical and surgical intensive care patients. Anaesthesia 2014;69:540-9.

28. Elliott R, McKinley S. The development of a clinical practice guideline to improve sleep in intensive care patients: a solution focused approach. Intensive Crit Care Nurs 2014;30:246-56.

29. Frisk U, Nordström G. Patients' sleep in an intensive care unit-patients' and nurses' perception. Intensive Crit Care Nurs 2003;19:342-9.

30. Krotsetis S, Richards KC, Behncke A, et al. The reliability of the German version of the Richards Campbell Sleep Questionnaire. Nurs Crit Care 2017;22:247-52.

31. Freedman NS, Gazendam J, Levan L, et al. Abnormal sleep/ wake cycles and the effect of environmental noise on sleep disruption in the intensive care unit. Am J Respir Crit Care Med 2001;163:451-7.

32. Salandin A, Arnold J, Kornadt O. Noise in an intensive care unit. $J$ Acoust Soc Am 2011;130:3754-60.

33. Simons KS, Verweij E, Lemmens PMC, et al. Noise in the intensive care unit and its influence on sleep quality: a multicenter observational study in Dutch intensive care units. Crit Care 2018;22:250.

34. Park M, Kohlrausch A, de Bruijn W, et al. Analysis of the soundscape in an intensive care unit based on the annotation of an audio recording. J Acoust Soc Am 2014;135:1875-86.

35. Darbyshire JL, Young JD. An investigation of sound levels on intensive care units with reference to the WHO guidelines. Crit Care 2013;17:R187.
36. Berglund B, Lindvall T, Schwela DH. World Health Organization Occupational and Environmental Health Team. Guidelines for community noise. 1999 http://www.who.int/iris/handle/10665/66217.

37. Celik S, Oztekin D, Akyolcu N, et al. Sleep disturbance: the patient care activities applied at the night shift in the intensive care unit. $J$ Clin Nurs 2005;14:102-6.

38. Pisani MA, Friese RS, Gehlbach BK, et al. Sleep in the intensive care unit. Am J Respir Crit Care Med 2015;191:731-8.

39. Cooper AB, Thornley KS, Young GB, et al. Sleep in critically ill patients requiring mechanical ventilation. Chest 2000;117:809-18.

40. Beecroft JM, Ward M, Younes M, et al. Sleep monitoring in the intensive care unit: comparison of nurse assessment, actigraphy and polysomnography. Intensive Care Med 2008;34:2076-83.

41. The Joint Commission. The Joint Commission announces 2014 national patient safety goal. 2016 http://www.jointcommission.org/ assets/1/18/jcp0713_announce_new_nspg.pdf

42. McKinley S, Fien M, Elliott R, et al. Sleep and psychological health during early recovery from critical illness: an observational study. $J$ Psychosom Res 2013;75:539-45.

43. Rotondi AJ, Chelluri L, Sirio C, et al. Patients' recollections of stressful experiences while receiving prolonged mechanical ventilation in an intensive care unit*. Crit Care Med 2002;30:746-52.

44. Ding Q, Redeker NS, Pisani MA, et al. Factors Influencing Patients' Sleep in the Intensive Care Unit: Perceptions of Patients and Clinical Staff. Am J Crit Care 2017;26:278-86.

45. Gabor JY, Cooper AB, Crombach SA, et al. Contribution of the intensive care unit environment to sleep disruption in mechanically ventilated patients and healthy subjects. Am J Respir Crit Care Med 2003:167:708-15

46. Perlo J, Balik B, Swensen S, et al. IHI framework for improving joy in work. Cambridge, MA: Institute for Healthcare Improvement, 2017.

47. Gandhi TK, Kaplan GS, Leape L, et al. Transforming concepts in patient safety: a progress report. BMJ Qual Saf 2018;27:1019-26. 